\title{
La escolarización interrumpida: perspectivas pedagógicas de educadores que trabajan con adolescentes bilingües refugiados en el programa RiseUp en los Estados Unidos de América
}

\author{
School Interrupted: Pedagogical perspectives of educators \\ working with bilingual adolescents refugees within the RiseUp \\ program in the United States
}

ISSN 1510-2432 - ISSN 1688-9304 (en línea) - DOI: https://doi.org/10.18861/cied.2018.9.2.2856

Lourdes Cardozo Gaibisso

Doctora (Cand.) en Educación, Lenguaje y Alfabetización, Universidad de Georgia (Estados Unidos). Investigadora en aprendizaje profesional docente, alfabetización científica para estudiantes bilingües y evaluación. Ha dictado cursos sobre adquisición del lenguaje, bilingüismo, aprendizaje profesional docente y liderazgo educativo en la Universidad de Georgia y en el Instituto de Educación de la Universidad ORT Uruguay.

Max Vázquez Domínguez

Doctor en Teoría y Práctica Educativa, Universidad de Georgia (Estados Unidos). Profesor e investigador, departamento de Educación de Ciencias, Universidad del Norte de Georgia (Estados Unidos). Ha trabajado con estudiantes latinos y sus familias, con maestros de ciencia y con profesores y estudiantes universitarios para mejorar los procesos de enseñanza y aprendizaje utilizando diferentes herramientas culturales como bilingüismo y fútbol.

Martha Allexsaht-Snider

Doctora en Educación Intercultural, Universidad de California (Estados Unidos). Profesora, departamento de Teoría y Práctica Educativa e investigadora sobre las interacciones familia-escuela-comunidad en diversos entornos, incluidas las comunidades latinas de Estados Unidos y de las áreas rurales de México,Universidad de Georgia (Estados Unidos).

Cory Buxton Doctor en Enseñanza y Curriculum con énfasis en Ciencias, Universidad de Colorado (Estados Unidos). Profesor, Universidad Estatal de Oregon (Estados Unidos). Investigador en oportunidades de aprendizaje de ciencias más equitativas para los estudiantes bilingües emergentes y en crear espacios donde estudiantes, padres, profesores e investigadores colaboran en la construcción de aprendizaje científico.

Fecha de recibido: 04/09/2018

Fecha de aceptado: 22/11/2018

\section{Resumen}

Un número considerable de estudiantes inmigrantes con escolarización interrumpida ha llegado a las aulas de enseñanza secundaria de algunas regiones de los Estados Unidos. En este contexto los educadores se ven ante el desafío de repensar sus prácticas de aula. Este estudio exploró cómo tres educadores -un profesor de aula, un profesor universitario y un asistente de investigación- perciben y narran la enseñanza bilingüe de la ciencia a un grupo de estudiantes refugiados en el sureste de los Estados Unidos. A través del Análisis Temático Crítico del Discurso este artículo abordó los discursos emergentes en 
los educadores sobre el conocimiento experiencial, el lenguaje cotidiano y el papel de la traducción en el aula de ciencias. Este estudio reveló la necesidad de conceptualizar la enseñanza de los estudiantes refugiados desde una perspectiva integral, que incluye no sólo aspectos pedagógicos sino también la necesidad de detectar y cuestionar discursos potencialmente deficitarios. Asimismo, este estudio propone reflexionar cómo cada sociedad, inscripta en un determinado momento histórico, político, cultural y económico en el que surge el saber académico genera las condiciones para que el mismo sea considerado importante y significativo. Nuestro papel como investigadores educativos es, entonces, desafiar y transformar estas condiciones para mejorar las oportunidades de aprendizaje de todos los estudiantes.

Palabras clave: estudiantes refugiados, bilingüismo, educación internacional, cambio educativo y demográfico, análisis crítico del discurso, enseñanza de la ciencia

\begin{abstract}
A significant number of immigrant students with interrupted schooling have arrived to secondary school classrooms in different parts of the United States. In this context, educators feel challenged to rethink their classroom practices. This study explored how three educators -a classroom teacher, a university professor and a research assistantperceived and narrated bilingual science teaching to a group of refugee students in the southeastern United States. Through Critical Thematic Discourse Analysis, this article addressed educators' evolving discourses on experiential knowledge, everyday language, and the role of translations in the classroom. This study revealed the need to conceptualize the teaching of refugee students from a holistic perspective, which includes not only pedagogical aspects but also the examination of potentially deficit discourses. Also, this study invites readers to reflect on how each society, embedded in a certain historical, political, cultural, and economic context where academic knowledge arises, generates the conditions for said knowledge to be considered relevant and meaningful. Our role then, as educational researchers, is to challenge and transform these conditions to improve learning opportunities for all students.
\end{abstract}

Key words: refugee students, bilingualism, international education, educational and demographic change, critical discourse analysis, science education

\title{
Objetivos
}

El cambio demográfico en el sureste de los Estados Unidos, explicado por una variedad de causas tales como el aumento de la demanda de las industrias manufactureras y agrícolas (Guzmán y McConnell, 2002) ha dado lugar a un aumento en el número de inmigrantes que llegan a los estados de esta zona del país; una región que, hasta fin del siglo pasado, permanecía relativamente estable en cuanto a los cambios étnicos (Portes y Salas, 2015). Sin embargo, un nuevo tipo de inmigración sacudió a los Estados Unidos en el año 2014 cuando una ola de niños sin la compañía de adultos -en su mayoría provenientes de Centroamérica- comenzó a ingresar al país a través de la frontera con México y no se contaba ni con recursos ni con una planificación estratégica para afrontar este fenómeno. Como resultado, durante el segundo semestre de 2014 el estado de Georgia comenzó a recibir un número creciente de estudiantes refugiados. Estos estudiantes, provenientes en su mayoría de América Central y México poseían una amplia diversidad en cuanto a antecedentes escolares, manejo de los idiomas inglés y español y conocimiento académico. Tras recibir este alto influjo de estudiantes refugiados, el distrito escolar objeto de estudio decidió crear un programa bilingüe que llamaremos el programa RiseUp. Este nuevo programa fue diseñado e implementado por un grupo de educadores del sistema escolar del condado en colaboración con profesores y estudiantes de doctorado de una de las universidades más importantes del estado. 
En lo referente al mismo deben destacarse los siguientes aspectos:

1) Los profesores y estudiantes de la universidad que formamos parte de este estudio hemos trabajado en grupo con estudiantes inmigrantes y sus familias -en su mayoría provenientes de México- en la educación de ciencia desde el año 2008.

2) El grupo de profesores y estudiantes de la universidad está conformado por personas bilingües y nuestro enfoque pedagógico fue también bilingüe.

3) El grupo de profesores y estudiantes de la universidad colaboró con docentes del sistema educativo.

Debido a que la situación de haber recibido en el estado de Georgia una gran cantidad de menores no acompañados es un fenómeno nuevo -aunque prevalente también en otros estados inmigrantes como California, Texas, Nueva York, Florida, Illinois y Arizona (OELA, 2008)- hay poca información sobre qué diseño curricular es el más adecuado para favorecer sus aprendizajes y, en consecuencia, no se conoce qué prácticas educativas benefician a estos estudiantes bilingües emergentes en este contexto particular. Como resultado, se han realizado muchos cambios en el programa en el que participamos durante cuatro semestres. Estas modificaciones tienen la característica primordial de que no pueden ser planificadas por completo debido a la poca experiencia con esta población de estudiantes por parte de las instituciones educativas y los maestros en servicio (Hamann y Harklau, 2010). Dado este contexto escolar dinámico y complejo es necesario repensar y rediseñar constantemente las prácticas docentes y crear espacios de aprendizaje profesional no sólo para los alumnos sino también para los profesores.

Debido a esta situación dinámica, nos planteamos los siguientes objetivos:

- Objetivo principal (en relación con los educadores participantes en este proyecto):

* Conocer cuáles son las perspectivas pedagógicas de los educadores de ciencia que trabajan con estudiantes refugiados en el programa RiseUp, y cómo estas perspectivas pueden contribuir a la alfabetización bilingüe en ciencia de dichos estudiantes.

- Objetivos secundarios (entre otros):

* Conocer cuáles son las perspectivas de los educadores acerca de las experiencias de los estudiantes y el valor de las mismas en el salón de clase cuando se enseña ciencia.

* Indagar acerca de cómo los educadores perciben la lengua materna de los estudiantes (español) y su rol en el aprendizaje del inglés y de la ciencia.

- Objetivo final:

* Reflexionar sobre las prácticas de enseñanza forjadas en el marco de la colaboración entre la escuela secundaria y la universidad.

Con estos objetivos en nuestra agenda de investigación aspiramos a que este estudio contribuya de manera más general a la investigación en el campo de la educación para inmigrantes latinos; un tema aún pendiente en la agenda de investigación internacional (Gándara, 2015). 


\section{Antecedentes}

\section{El fenómeno migratorio}

La migración desde países como México y regiones como Centroamérica y Sudamérica a los Estados Unidos es un fenómeno que continúa siendo importante en el siglo XXI. Sin embargo, a principios del presente siglo comenzó a darse una migración particularmente diferente a las anteriores ya que niños de México, El Salvador, Guatemala y Honduras emprendieron su huida hacia los Estados Unidos debido a la violencia prevalente en sus regiones de origen. Aunque hay registros de la migración de niños a los Estados Unidos ya desde el año 2008, este tipo de migración empezó a ser notablemente significativa a partir del año 2012 (Seghetti, Siskin y Wasem, 2015). En este contexto el gobierno de los Estados Unidos, bajo el mandato del presidente Barack Obama, declaró una crisis humanitaria ante la incapacidad del estado para enfrentar esta situación (Department of Justice, 2014). Por una parte, en el año 2012 y como parte de la respuesta a la crisis humanitaria, el gobierno de los Estados Unidos realizó acciones para controlar la frontera y prevenir el ingreso de la juventud migrante. Por otra parte también hubo intentos por ayudar a estos niños y reunirlos con sus familiares en los Estados Unidos. Las organizaciones gubernamentales que están a cargo de los procesos migratorios de estos niños primordialmente son el Departamento de Seguridad Nacional (Department of Homeland Security, DHS) y la Oficina de Reasentamiento de Refugiados (Office of Refugee Resettlement, ORR) (Administration for Children \& Families, 2017). Ambas oficinas gubernamentales tienen diferentes objetivos y obtienen recursos económicos muy desiguales para apoyar sus causas. Por ejemplo, por un lado, en el año fiscal 2015 la Oficina de Reasentamiento de Refugiados, que es la responsable de cuidar y supervisar el cumplimiento de los derechos de los niños, obtuvo de la federación 1600 millones de dólares para su financiamiento. Por otro lado, se otorgaron 3400 millones de dólares para el Departamento de Seguridad Nacional (Kandel, 2017). Lo que sucede luego de que los migrantes cruzan la frontera es complejo, y lo describiremos a continuación.

Inmediatamente después de que el Departamento de Seguridad Nacional detiene a los niños en la frontera y recopila toda su información los trasfiere a la Oficina de Reasentamiento de Refugiados. El primer paso -la detención- es un procedimiento en el cual la DHS (Departamento de Seguridad Nacional de los Estados Unidos) se asegura de proteger a esta población a la vez que se cerciora de que se dirijan a los juzgados donde cada uno de ellos deberá defender su situación ante un juez de migración (Terrio, 2015). Es importante mencionar que estos niños son vistos legalmente como adultos en estos tribunales. Por tal motivo, los mismos son juzgados bajo las mismas normas a las que están sujetas las personas mayores de edad. Aunado a esta desventaja de ser requerido a presentarse legalmente como adulto en los tribunales, los niños no obtienen ninguna ayuda de abogados para defenderse por vía del estado. El proceso legal de cada uno de estos niños es individual y mientras las audiencias se programan para establecer los procedimientos legales, la ORR se encarga de asignarles un patrocinador que es, habitualmente, un familiar que reside en los Estados Unidos (Administration of Children and Families, 2017).

En el estado de Georgia, en el año 2014 hubo 1412 casos registrados de los cuales 28 fueron realojados en el condado escolar donde tuvo lugar esta investigación (WSBTV, 2014). Al entrevistar a los estudiantes para asignarles el grado escolar y su salón de clases los maestros se dieron cuenta de que las experiencias en educación formal en los sistemas educativos de sus países de procedencia eran muy diversas. La realidad mostraba que muchos de estos estudiantes habían experimentado una escolarización interrumpida. Hubo casos en los que su paso por la educación formal fue inexistente y hubo otros en los que la misma quedó trunca (Terrio, 2015). Esta heterogeneidad en la escolaridad de los niños migrantes ha añadido más dificultades al proceso de adaptación social y dio paso a diversas propuestas que describiremos brevemente a continuación. 
Históricamente, la educación bilingüe en escuelas públicas en Georgia ha sido prácticamente nula y las herramientas que los maestros obtienen durante su formación en las universidades es muy limitada. Esto, en parte, se debe a las iniciativas que favorecen la enseñanza y uso exclusivo del idioma inglés en las escuelas públicas (Davis, 2000). Sin embargo, grupos de maestros en las instituciones educativas, profesores universitarios, estudiantes de posgrado y personal de las escuelas han tratado de mejorar la situación educativa de estos niños migrantes al formar redes comunitarias para aportar, utilizar y diseñar herramientas que les sirvan para su adaptación en este nuevo contexto social. Esta investigación se enmarca en esas nuevas experiencias académico-bilingües que tienen como aspiración llenar un persistente vacío curricular e institucional.

\subsection{Programas para estudiantes inmigrantes e introducción del programa RiseUP}

El programa RiseUp surge de la necesidad de dar atención educativa a un grupo de estudiantes inmigrantes recién llegados al estado de Georgia. Tal como se mencionó anteriormente el programa se desarrolla en una escuela chárter que funciona como una preparatoria y que brinda a los estudiantes la posibilidad de graduarse en una carrera técnica en diferentes áreas tales como gastronomía, hotelería, negocios o mercadotecnia. Sin embargo se estableció como un programa que sigue el modelo de instrucción de refugio. Es decir, la mayoría de los estudiantes en RiseUp no tomaron las clases que la preparatoria ofrece a los alumnos regulares. Las clases de RiseUp se diseñaron basándose en las características de los alumnos refugiados, con las herramientas, materiales y recursos disponibles y en base al conocimiento acerca de la elaboración de planes educativos para refugiados así como a la ayuda y experiencia de los profesores y alumnos de la universidad que participaron en este programa.

En nuestra experiencia en el estado de Georgia, los programas más comúnmente utilizados en las escuelas de educación básica con estudiantes inmigrantes consisten en una estructura llamada Pull-out. Esta práctica consiste en retirar a los estudiantes del aula regular donde todos los alumnos toman sus clases, para que reciban apoyo en clases de inglés en forma individual o en grupos pequeños (Fernández y Hynes, 2016). En el caso del programa RiseUp, los estudiantes fueron agrupados fuera de las clases regulares ya que el contenido curricular fue cualitativa y cuantitativamente diferente. El criterio utilizado para la formación de los grupos en el programa fue, principalmente, el de la edad, el conocimiento del idioma inglés y la facilidad para expresarse en dicha lengua.

Por una parte, como investigadores y educadores hemos encontrado grandes limitaciones en la literatura sobre escolarización interrumpida y programas para refugiados. Hunkapiller (2010), por ejemplo, ha sido uno de los pocos autores que ha abordado este concepto tomando en consideración las brechas en la educación de estos estudiantes en sus países de origen. Por otra parte, tradicionalmente, estos programas se ubican por separado en otras escuelas y atienden únicamente a estudiantes inmigrantes (Feinberg, 2000).

En nuestro caso, el programa para recién llegados se ubica dentro de una escuela chárter y funciona como un modelo de instrucción de refugio. Es decir, los estudiantes sólo toman su clase de ESOL (English for Speakers of Other Languages - Inglés para hablantes de otras lenguas) y la clase de ciencias, en este caso, con otros jóvenes inmigrantes recién llegados al país. Las clases de ESOL y las clases de las asignaturas regulares, como la de ciencia, fueron diseñadas partiendo de una evaluación diagnóstica.

A principios de 2014, a partir de la interacción en el aula con un número de aproximadamente treinta estudiantes refugiados supimos que sus lugares de procedencia eran heterogéneos. Los mismos provenían de zonas rurales, suburbanas y urbanas. Muchos estudiantes recién llegados habían tenido una educación interrumpida mientras que otros no habían recibido ninguna formación ni acceso a la educación formal en absoluto. Esto depende de la diversidad de recursos que los países de procedencia asignan a la educación en general, así como a los recursos y al énfasis que cada maestro destina a sus estudiantes. 
Respecto al conocimiento del idioma inglés de los alumnos del programa RiseUp había heterogeneidad en lo que refiere a sus capacidades para hablar, comprender, leer y escribir dicha lengua. El nivel de conocimiento del inglés se corresponde con el poco énfasis que los diferentes países de Latinoamérica destinan a su enseñanza (Cronquist y Fiszbein, 2017). Este fue un aspecto que resaltamos en nuestra enseñanza y en el diseño de actividades en el salón de clases.

En cuanto a las interacciones hemos notado, tal como mencionan Patel, Tabb, Strambler y Eltareb (2015), que el nuevo escenario cultural suele presentar un gran desafío para todos los involucrados. Como resultado, y como forma de paliar este brusco cambio, las escuelas ubicadas en regiones geográficas que reciben jóvenes recién llegados se enfrentan a la tarea de diseñar programas que puedan abordar este desafío junto a los estudiantes. Las preguntas clave que como educadores e investigadores debemos plantearnos son: ¿Quién específicamente debe hacer eso? ¿Los docentes? ¿Los especialistas de los programas de estudio? ¿Quién tiene tiempo y pericia para hacer este trabajo? Este artículo pretende mostrar tan solo un ejemplo de la colaboración de diversos actores de la comunidad educativa que trabajaron para alcanzar dicho fin.

\section{Enseñanza de estudiantes lingüística y culturalmente diversos}

En la sección anterior describimos algunas características generales de los estudiantes inmigrantes y cómo se conceptualiza un programa para recién llegados. Los docentes, sin duda, tienen un fuerte impacto en la vida de sus estudiantes y las poblaciones inmigrantes no son una excepción. En este sentido, los docentes de esta población estudiantil tienen la oportunidad de utilizar las experiencias previas y expectativas futuras de sus estudiantes e integrarlas de manera significativa a los contenidos curriculares (Oikonomidoy, 2014).

Al pensar en las experiencias de escolarización de los estudiantes recién llegados es necesario reconocer cuáles son los vacíos en la formación docente. Durante más de una década los investigadores han argumentado que la noción de la formación de docentes especializados (por ejemplo, las certificaciones de docentes de ESOL) son obsoletas en un clima cada vez más lingüística y culturalmente diverso. La literatura actual (Gort, Glenn y Settlage, 2010; De Jong y Harper, 2011; Lucas y Villegas, 2011) confirma que los programas de compartimentación de formación docente obstaculizan las oportunidades de aprendizaje académico de los estudiantes pertenecientes a minorías lingüísticas y culturales y refuerzan las prácticas hegemónicas. Asimismo, la diversificación de los candidatos docentes es crucial (Sleeter, 2001). Sin embargo, debido a que la gran mayoría de los candidatos a docentes y administradores escolares en los Estados Unidos son blancos y monolingües existe una necesidad de crear políticas educativas que se enfoquen en diversificar prácticas lingüísticas que cuestionen las estructuras escolares que marginan a los estudiantes inmigrantes.

Si bien deben abordarse estos temas en la formación inicial de docentes, los estudiantes recién llegados ya están asistiendo a las escuelas en la actualidad y cada distrito escolar debe asegurar que reciban una educación de calidad. Las asociaciones y vínculos colaborativos entre la universidad y la escuela, así como los modelos de desarrollo profesional docente pueden servir como un medio para abordar este escenario complejo y mejorar las experiencias de aprendizaje de los estudiantes inmigrantes (Gooden y Chase, 2015).

Dada nuestra experiencia previa de trabajo con estudiantes latinos y sus familias en el sistema educativo de la región donde el programa RiseUp se desarrolla, la escuela nos contactó para apoyarlos con el diseño y la implementación del programa con estudiantes refugiados. 
Nuestra experiencia como grupo de investigación en la enseñanza de la ciencia comenzó en el año 2008. Desde ese año nuestro trabajo ha estado enfocado en los estudiantes latinos y sus familias siguiendo como criterio principal el abordaje de la necesidad imperante de colaborar con los maestros de ciencia en el diseño de estrategias docentes que usen herramientas culturales como objetos esenciales en la enseñanza de las ciencias y, por lo tanto, la utilización del español y otros recursos que forman parte del bagaje de estas familias y estudiantes en el salón de clase.

\section{La importancia de la ciencia y las claves para el aprendizaje del lenguaje científico}

Así como hay organizaciones en educación que enfatizan la importancia de aprender ciencia a nivel internacional (por ejemplo, la Organización de Estados Iberoamericanos) y dentro de los Estados Unidos (por ejemplo, la National Science Teachers Association y la National Academy Press), también nosotros como educadores creemos que es necesario promover una educación en ciencia, en nuestro caso, con los estudiantes refugiados en los Estados Unidos. Las razones por las que creemos que es necesario enseñar ciencias también coinciden con lo afirmado por Kinslow y Sadler (2018) quienes mencionan diferentes motivos que van desde los educativos, como la importancia de promover pensamiento crítico y complejo, hasta los sociales, como la creciente falta de profesionales en el campo de la ciencia para cubrir las demandas sociales en salud, bioingeniería e ingeniería y cambio climático, entre otras áreas.

Sin embargo, al tratar de alinear los objetivos de enseñanza de la ciencia al grupo heterogéneo de estudiantes refugiados tomamos conciencia del gran desafío que representa adaptar las actividades curriculares al bagaje cultural de los estudiantes refugiados y las experiencias que se generan como educadores en estos ámbitos educativos inéditos en la región.

Los estudiantes recién llegados cuyo idioma materno no es el inglés, y que han sufrido con frecuencia experiencias escolares interrumpidas, se enfrentan a la tarea de aprender, simultáneamente, un segundo idioma y el conocimiento vinculado a cada asignatura. Asimismo, estos estudiantes deben adquirir los elementos léxicos y técnicos específicos de cada área de contenido (Callahan y Shifrer, 2016). Uno de los contenidos más desafiantes es la ciencia ya que los estudiantes necesitan desarrollar sus habilidades de aprendizaje científico y también dominar los elementos léxicos y gramaticales del Lenguaje de la Ciencia (LDC). Una vez que los estudiantes han desarrollado herramientas para comprender y producir este lenguaje la comprensión se facilita pero, hasta que no lo logren, los contenidos tendrán poco o ningún sentido para ellos (Lemke, 1990).

De manera similar Halliday (2004) argumenta que uno de los principales desafíos para aprender LDC no está exclusivamente vinculado a la densidad del vocabulario altamente técnico sino a su estructura léxico-gramatical altamente compleja. Tomando en cuenta la complejidad del LDC, las características de los estudiantes recién llegados, las herramientas disponibles para la enseñanza de la ciencia en estos contextos y la experiencia de los maestros en este ámbito se necesita de un conjunto de teorías y metodologías que puedan estudiar, explicar y analizar esta problemática para dar un conjunto de posibles soluciones. 


\section{Marco Teórico}

Este trabajo se enmarca en los principales conceptos relacionados con la Teoría Crítica y se apoya en el Análisis Crítico del Discurso como herramienta teórico-analítica. La teoría social crítica puede definirse como un elemento orientador que provee a los investigadores y educadores de herramientas de reflexión crítica acerca de cómo su praxis se relaciona con estructuras políticas, económicas y sociales (Freeman y Vasconcelos, 2013).

En el contexto de este estudio la Teoría Crítica se concibe como un término amplio para el cual diferentes lentes críticos se enfocarán en una diversidad de temas sociales, no con la única intención de describir o explicar sino con un marcado propósito de producir cambios sociales mediante el cuestionamiento y la transformación de estructuras dominantes.

El marco conceptual de este estudio se basa en lo que consideramos las prolíficas y enriquecedoras intersecciones entre la Teoría Crítica y un enfoque crítico del lenguaje conocido como Análisis Crítico del Discurso (Rogers, 2011).

A continuación se establece la forma en la que se utilizó la Teoría Crítica y el Análisis Crítico del Discurso para responder a las preguntas de investigación planteadas.

El Análisis Crítico del Discurso, anclado en la Teoría Crítica, proporciona las herramientas para abordar la complejidad de las prácticas discursivas en los centros educativos, ayudando a entender las prácticas dentro de instituciones donde las desigualdades son frecuentes. En un sentido amplio, las teorías sociales críticas junto al Análisis Crítico del Discurso pueden ayudar a educadores e investigadores a responder preguntas de investigación particulares (Rogers, 2011). En este sentido, el Análisis Crítico del Discurso está basado en diferentes disciplinas y tiene por objetivo descubrir y hacer explícitamente visible la conexión entre lenguaje y poder y, como consecuencia, el lenguaje como un medio a través del cual se legitima la cultura hegemónica.

En esta dirección se aleja de la idea del discurso como un acto neutral, considerando su impacto en múltiples contextos, incluido el educativo. Por tal motivo el Análisis Crítico del Discurso tiene un interés particular en comprender la relación entre lenguaje y poder (Wodak, 2005). Asimismo, puede ayudar a los educadores a reflexionar críticamente sobre las prácticas y discursos que están presentes en sus aulas, en sus escuelas y en el sistema educativo en general. También permite a los educadores desafiar los discursos dominantes sobre ellos mismos y sus estudiantes. Esta combinación puede llegar a ser especialmente relevante para los estudiantes sistemáticamente vulnerados, como es el caso de los inmigrantes y refugiados. En el caso de estos estudiantes -población objeto del presente estudio- crea un espacio para reimaginar y crear espacios alternativos que desmantelan los discursos dominantes y discriminatorios a los que los estudiantes están expuestos.

\section{Propósito y preguntas de investigación}

Como hemos establecido en secciones previas de este artículo, a medida que el número de estudiantes recién llegados continúa creciendo los educadores tienen la obligación y el desafío de repensar sus prácticas de enseñanza. Los docentes, especialmente en aquellos estados donde la inmigración es un fenómeno nuevo, generalmente tienen dificultades para reconocer el valor del conocimiento experiencial adquirido en otros entornos no formales que los estudiantes no dominantes traen al aula. A medida que estos estudiantes navegan el sistema escolar estadounidense es imperante que vivan experiencias de aprendizaje significativas que incluyan el reconocimiento de la conexión del contenido académico con sus recursos lingüísticos y culturales (Cardozo Gaibisso, Allexsaht-Snider y Buxton, 2017). Por tal motivo realizamos un análisis temático de entrevistas en profundidad con tres educadores que desde octubre de 2014 han venido trabajando con los estudiantes recién llegados. A través de estas entrevistas indagamos acerca de cómo estos docentes de 
ciencias perciben el papel de los conocimientos previos experienciales de los estudiantes, ayudándolos a dar sentido al contenido y al lenguaje que se usa para comunicarlo. Fuimos observando los patrones temáticos a medida que surgían en las entrevistas. Finalmente examinamos las perspectivas de los educadores sobre el papel de la lengua materna de los estudiantes (español) en el aprendizaje del inglés como segundo idioma (ESL, English as a Second Language - Inglés como Segunda Lengua) y la ciencia.

Este estudio se propone analizar datos recopilados de este modelo de instrucción científica para estudiantes bilingües emergentes en esta escuela en particular. Según los entrevistados, los estudiantes bilingües recién llegados se sumergieron en un modelo de instrucción de ciencias que aprovechó, desde el punto de vista pedagógico, su repertorio lingüístico y sus experiencias para aprender tanto el contenido de ciencias como el lenguaje. Las preguntas de investigación fueron las siguientes:

1) ¿Cómo perciben estos educadores el conocimiento previo y experiencial de los estudiantes y cómo se relaciona con la puesta en marcha del Programa RiseUp?

2) ¿Cuáles son las perspectivas de los educadores sobre el papel de la lengua materna de los estudiantes (español) en el aprendizaje del inglés como segundo idioma (ESL) y la ciencia?

\section{Métodos}

Según Cameron (2001) el uso del lenguaje es una práctica social que ayuda a los individuos a construir sus propias identidades y plasmar las relaciones de poder. En este contexto, el discurso puede definirse como una forma de explorar cómo se construyen y expresan estas relaciones. El poder se puede situar dentro de los discursos, y hay contextos en los cuales el desequilibrio del poder puede ser identificado más fácilmente; tal es el caso de las interacciones entre profesores y estudiantes. Son las interacciones profesor-alumno que se dieron en el programa RiseUp durante el segundo semestre del año 2016 las que estudiamos en esta investigación usando como metodología el Análisis Temático Crítico del Discurso. A través de este enfoque metodológico analizamos tres entrevistas semiestructuradas con el fin de responder a las preguntas de investigación planteadas en la sección previa bajo la premisa de que el uso del lenguaje es una parte primordial de la vida y de las experiencias sociales.

El valor del Análisis Temático Crítico radica en que es una herramienta de investigación que nos permite localizar y estudiar las relaciones sociales de poder en el discurso y así tener un sustento para poder actuar y permitir un cambio pedagógico. Conjuntamente utilizamos entrevistas semiestructuradas que enmarcan la temática de la educación a refugiados en el programa RiseUp en los Estados Unidos. Cuando los participantes describieron sus experiencias dando clases en el programa RiseUp, estas metodologías nos permitieron analizar en cada uno de los discursos cómo cada participante construyó significados de acuerdo a las experiencias con los alumnos, el lugar, los colegas y las creencias y presupuestos culturales en que se basaron.

\section{Participantes}

Los participantes de este estudio fueron tres docentes que trabajaron en el Programa RiseUp con un grupo de estudiantes recién llegados desde octubre de 2014 hasta abril de 2016. Como resultado de una asociación universidad-escuela, una profesora universitaria bilingüe (Michelle) nacida en los Estados Unidos, un asistente de investigación bilingüe (Pedro) nacido en México y una maestra de ciencias nacida en los Estados Unidos (Sally) trabajaron juntos en diferentes contextos: cooperando y enseñando en el programa 
RiseUp, participando en sesiones de aprendizaje profesional y asistiendo a un instituto de docentes de verano. Todas esas experiencias han informado sus prácticas y percepciones sobre el trabajo con estos grupos de estudiantes.

Tabla 1. Información demográfica de los participantes

\begin{tabular}{|l|l|l|l|l|}
\hline & Género & País de origen & $\begin{array}{l}\text { Rol dentro de la } \\
\text { escuela }\end{array}$ & Lenguaje nativo \\
\hline Sally & Femenino & Estados Unidos & Maestra & Inglés \\
\hline Pedro & Masculino & México & $\begin{array}{l}\text { Asistente de } \\
\text { Investigación }\end{array}$ & Español \\
\hline Michelle & Femenino & Estados Unidos & $\begin{array}{l}\text { Profesora } \\
\text { Universitaria }\end{array}$ & Inglés \\
\hline
\end{tabular}

Michelle y Pedro asistieron a la escuela y enseñaron en el Programa RiseUp una vez a la semana durante un total de cuatro semestres. Sally estuvo presente en el aula la mayor parte del tiempo y ocasionalmente se involucraba con la lección o abordaba cuestiones disciplinarias. Sin embargo, los días en que el equipo de la universidad visitó el programa RiseUp sus integrantes fueron quienes llevaron adelante la mayor parte de la instrucción pedagógica en el aula.

\section{Recopilación de datos}

Este estudio consiste en tres entrevistas semiestructuradas que se llevaron a cabo durante el primer semestre del año 2016. La guía de entrevistas se desarrolló con el objetivo de reunir una amplia narrativa de las perspectivas de los educadores sobre sus prácticas de enseñanza. La maestra del aula, la profesora universitaria y el asistente de investigación trabajaron con los estudiantes recién llegados en el programa RiseUp desde octubre de 2014 hasta abril de 2016.

\section{Análisis de datos}

Para el análisis de los datos se realizó un Análisis Crítico del Discurso buscando pautas clave que emergieran de las entrevistas (Ruona, 2005). Conjuntamente, se buscó situar críticamente las perspectivas de los educadores en contextos sociales más amplios. El Análisis Crítico del Discurso como enfoque analítico de las entrevistas busca descomprimir datos basados en una perspectiva crítica y situacional de temas emergentes (Gee, 2015). A través de este enfoque se codificaron y analizaron las tres entrevistas. Por último, se interpretaron de manera crítica las narraciones de los participantes atendiendo al contexto institucional y social. En este sentido, describir y analizar los discursos de educadores se transforma en un recurso que nos permite redescubrir y reconceptualizar el rol de los estudiantes que han sido tradicionalmente marginados y cuyo conocimiento y experiencia no han sido tomados en cuenta en los procesos de enseñanza y aprendizaje.

\section{Resultados}

\section{Temas emergentes}

En esta sección presentamos los principales hallazgos que surgen de las entrevistas realizadas a los educadores que participaron en la investigación. Entre los temas emergentes se encontraron tres principales que se describen a continuación:

La construcción de relaciones interpersonales a través de la lengua hablada en el hogar de los estudiantes 
Un tema común entre los participantes fue la noción de utilizar los idiomas del hogar de los estudiantes, en este caso el español, para mejorar la construcción de relaciones interpersonales entre los miembros de la comunidad educativa. Las narrativas de los entrevistados muestran una tendencia a entender el español como un recurso fundamental para construir un clima respetuoso y afectuoso en el aula.

En este sentido, Sally, afirma:

"Mi aprendizaje [de español] ha sido solo de supervivencia, realmente lo quiero, es importante para mí que entiendan que estoy tratando de entenderlos, y creo que para enseñar y... construir la relación y la confianza que necesitan \{para\} sobresalir necesitan saber que estoy trabajando duro para encontrarlos donde están y creo que ese ha sido el factor motivador detrás de esto. Es que quiero que vean que no es fácil \{aprender otro idioma\} así que me parece genial ver que me veo como una idiota y está bien (se ríe). Sobreviví".

Sally, la maestra que comparte gran parte del tiempo pedagógico semanal con los estudiantes manifiesta que su deseo y motivación para aprender el español como segunda lengua no solo se basa en la construcción de vínculos sino en la aspiración de servir como modelo de estudiantes de una segunda lengua. De esta forma Sally rompe con el estereotipo dentro de las escuelas estadounidenses que sostiene que el uso exclusivo del inglés debe ser el único foco en el salón de clases.

Siguiendo esta idea y en respuesta a la pregunta sobre cómo piensa que el conocimiento de la lengua materna de los estudiantes influye en el proceso de enseñanza y aprendizaje, Pedro sostiene que:

“Creo que es primordial, es muy importante y lo puedes notar. Si soy el estudiante y la maestra está aprendiendo algo que yo sé eso muestra algún tipo de respeto o algún tipo de interés, como que está demostrando que ella se preocupa por mí y si soy un estudiante eso es importante, eso es en términos de... del valor del estudiante y el maestro, pero en términos académicos creo que eso también es muy importante porque estás usando... eres un andamio."

Michelle, la profesora universitaria, también destacó el valor del uso del español en un aula compuesta por estudiantes inmigrantes, tanto para los profesores como para los alumnos:

"Creo que en un nivel muy fundamental fomenta la construcción de relaciones, especialmente importante cuando los estudiantes son nuevos en el entorno, en el contexto, nuevos en el idioma inglés y especialmente importante cuando los estudiantes pueden haber experimentado un trauma \{para\} llegar a \{la\} escuela, y llegar a este nuevo contexto, así que incluso poder hablar el idioma de los estudiantes rudimentariamente y hacer un esfuerzo para aprender. Creo que es importante en términos de construcción de relaciones... y demostrar que también eres un aprendiz del idioma. Me parece absolutamente crítico en términos de modelar el proceso de aprendizaje... también eres un estudiante de idiomas y reconoces lo que funciona en el proceso de aprendizaje del idioma porque también atraviesas ese proceso".

En esta sección sobre el uso de la lengua materna son varios los elementos que los entrevistados destacan. La valoración del español como medio para generar cercanía entre los estudiantes y, al mismo tiempo, modelar técnicas de aprendizaje, está presente en los discursos. En la sección que sigue mostraremos cómo los educadores, dando un paso más en su compromiso con los estudiantes, deciden repensar su praxis pedagógica a la luz de los recursos y repertorios lingüísticos disponibles para propiciar el aprendizaje. 
El aprovechamiento pedagógico de todos los repertorios y dominios lingüísticos de los estudiantes como pieza central de los procesos de aprendizaje de ciencias

Investigaciones recientes sugieren que utilizar los recursos lingüísticos de los estudiantes, por ejemplo sus idiomas de origen, las expresiones cotidianas y el vocabulario académico (García y Kleifgen, 2012) es un componente crucial para mejorar la comprensión del contenido académico de estudiantes bilingües. Los relatos de Michelle y Pedro revelaron una planificación e integración cuidadosas y proyectadas de esos repertorios y dominios en su enseñanza.

Michelle indica que:

“Trabajar con los estudiantes de RiseUP fue solo una especie de llamada de atención para reconsiderar... cómo andamiar y apoyar realmente a los \{estudiantes\} bilingües emergentes que son nuevos en inglés y realmente hablan muy poco o nada de inglés. Por lo tanto, ¿cómo puede la lengua materna ser un componente de la cadena para facilitar el aprendizaje continuo del contenido y del lenguaje académico en la escuela, al mismo tiempo que desarrolla las habilidades del inglés? No postergar la enseñanza del contenido y la comprensión del lenguaje académico relacionado con la ciencia hasta que los estudiantes hayan desarrollado más sus habilidades del idioma inglés".

Aquí Michelle cuestiona lo que convencionalmente se practica en las escuelas de los Estados Unidos: esperar a que los estudiantes que no hablan inglés de forma fluida aprendan el idioma para luego enseñar contenidos. Esta práctica ha causado rezago en los aprendizajes y ha impactado de forma negativa en el desarrollo académico de los estudiantes cuya lengua materna no es el inglés.

Pedro narra y elabora de manera más explícita lo que él llama el proceso de andamiaje en el lenguaje:

\begin{abstract}
"Al comienzo del programa hablamos español la mayor parte del tiempo y tratamos de complementar el español, usando un poco de inglés. Fue desafiante para algunos de ellos y ayudó a muchos estudiantes, pero algunos otros cayeron en una posición desventajosa porque no podían entender lo que el maestro estaba diciendo \{en inglés\}. Fue un desafío. Necesitas conocer el estado actual de los estudiantes en materia educativa y desde esa posición se necesita andamiar o llevar a la zona de máximo desarrollo académico. Para que ellos puedan comenzar a usar el lenguaje académico, el lenguaje cotidiano es muy enriquecedor".
\end{abstract}

Y agrega:

“Es un proceso continuo... Participar en diferentes actividades y desarrollar su conocimiento sobre sus nuevos objetivos, su uso del idioma, sus antecedentes y el nuevo entorno en el que se encuentran, por lo tanto, inglés y español, y sus metas en la vida, la institución académica, los objetivos institucionales".

Sally, a pesar de que reconoce su importancia, lo percibe como un desafío. Ella expresa sus dudas sobre qué es mejor enseñar primero, si el idioma -en este caso inglés- o el contenido. También menciona cómo los administradores escolares influyen en esta decisión: 
“Esa es una reconciliación muy difícil, porque aún estoy tratando de resolverlo, y como en años anteriores con el otro director \{la enseñanza\} estaba más basada en conceptos y con este nuevo director está basado más en el \{inglés\}... No sé. Para ser honesta, como maestra quiero que entiendan el concepto porque es más fácil para mí, y tengo un objetivo en mente sobre adónde quiero que lleguen, por eso es más fácil para mí usar el español, para asegurarme de que aprendan el concepto en lugar de tomar más tiempo y \{causar\} más frustración... De todos modos se convierte en una batalla para el maestro".

Y continúa:

“Voy a tomar el camino más fácil o voy a tomar el camino en que hay mucha... no necesariamente oposición, pero es una manera más difícil, así que no, sinceramente, no tengo la respuesta para eso porque todavía es una batalla en mi mente sobre qué es lo mejor... Así que tengo que tenerlo siempre en cuenta ¿Cuál es el objetivo? ¿Es inglés o es que necesito que entiendan qué es esto \{un concepto\}, y si lo hago: es importante que yo hable en español?"

El conocimiento experiencial de los estudiantes como punto de partida para enseñar ciencia Los tres entrevistados reconocieron el valor de incorporar el conocimiento y las experiencias previas de los estudiantes en su enseñanza de la ciencia. Revelaron usos explícitos de este conocimiento como medio de andamiar el proceso de aprendizaje. Sally afirma que, si bien no todas las áreas de contenido de ciencia son adecuadas para usar el conocimiento experimental, algunas de ellas son clave:

“En ciencias ambientales pude mucho porque... incluso en los volcanes, sabes, cosas con las que tenían experiencia... incluso la diferencia entre los sistemas de basura aquí y los que experimentan en su país de origen, entonces la ciencia ambiental fue una transición fácil en cuanto a la ciencia. La biología era una historia diferente, tiene un vocabulario diferente en sí mismo que es otro idioma".

Sally identifica las ciencias ambientales como una forma de conectar el conocimiento. Lo contrasta con el estudio de la biología que requiere que los estudiantes conozcan y entiendan vocabulario más complejo. Los estudiantes parecían tener menos experiencias para conectarlas con los temas a enseñar.

Pedro expresa una comprensión detallada de cómo los estudiantes ya poseían conocimiento sobre la ciencia, no por haber tenido contacto con esta área del conocimiento desde una perspectiva formal sino porque lo han experimentado en su vida cotidiana, antes de buscar refugio en los Estados Unidos. De todas formas, Pedro realiza una clara distinción entre conocer y experimentar la ciencia en la vida cotidiana, y poder expresarse y entender el lenguaje científico.

"Sabían de la ciencia, lo que no sabían era el lenguaje de la ciencia. Todos cocinaron una comida, todos usaron automóviles, por ejemplo, pero la cuestión es usar esa perspectiva científica para hablar sobre lo que está sucediendo en sus vidas. Ese es el desafío. Algunos de ellos, muy pocos, tenían algún conocimiento sobre algunos conceptos, hipótesis, causa y efecto y la mayoría no, a pesar de que habían experimentado la ciencia en sus vidas".

Michelle usa un ejemplo de una lección para explicar cómo conectó el aprendizaje de los estudiantes con el nuevo contenido de ciencia a ser enseñado: 
"Voy a pensar en las lecciones que hice sobre la medición, la construcción y las fracciones. Creo que es un buen ejemplo. Comencé diciendo: ‘Conocen a alguien que haya trabajado en la construcción? ¿Qué experiencia has tenido construyendo cosas? Cuándo construiste cosas ¿qué tipo de herramientas usaste?' Yo traje algunas fotos y algunos ejemplos de cosas y herramientas de medición, en unidades métricas, en pies y pulgadas para que los estudiantes comenzaran a pensar en eso, en su experiencia, cómo se aplica a la situación... y luego pensando en las fracciones... midiendo instrumentos y hablando de dónde y cómo se representaron las fracciones en esos instrumentos. Así que creo que es un ejemplo de cómo siempre estaba tratando de hacer eso \{conectar los nuevos contenidos, con las experiencias ya vividas por los estudiantes\}".

\section{Discusión de Resultados}

El propósito de este estudio ha sido explorar la perspectiva pedagógica de tres educadores en relación a cómo conceptualizan y comprenden su praxis educativa enfocada en los estudiantes refugiados. Esta temática, explorada en nuestro estudio en 2016, se vuelve aún más relevante en 2018 no solo en el contexto de los Estados Unidos sino en el contexto mundial (Cardozo Gaibisso, Allexsaht-Snider y Buxton, 2017).

Como se muestra en la sección anterior parece haber una comprensión común de las temáticas, que trascienden los roles individuales de los entrevistados. Una parte sustancial de los discursos de los participantes sobre su enseñanza muestra percepciones similares sobre la enseñanza de los estudiantes refugiados. El papel de la lengua materna de los estudiantes y las experiencias anteriores vinculadas a la ciencia fueron temas fundamentales a lo largo de las entrevistas.

Podemos agrupar las similitudes expresadas por los tres educadores del programa RiseUp de acuerdo a los siguientes puntos:

A) El diseño de planes de clase que requieran que los estudiantes apliquen sus experiencias científicas y conocimientos previos para explorar nuevos conceptos en inglés y en español. B) La identificación por parte de los maestros de un modelo flexible que sirva como medio de transición y andamiaje para la adaptación educativa, cultural y lingüística de los estudiantes refugiados y/o inmigrantes.

C) La relevancia tanto de la enseñanza y el aprendizaje en todas las lenguas habladas por los alumnos como de un modelo pedagógico dinámico que promueva una mayor comprensión conceptual de las ciencias.

Estos resultados muestran que, si bien el grupo de educadores tiene una noción clara de estos conceptos y su aplicabilidad, lo ideal sería contar con un sistema educativo que apoyara con los recursos humanos y materiales a las instituciones educativas y a los educadores como parte fundamental del sistema educativo. De esta forma, los educadores podrían comenzar a realizar pequeñas acciones dentro de su salón de clases para fomentar el proceso de enseñanza y aprendizaje de los alumnos refugiados en la enseñanza de la ciencia.

Para estudiantes recién llegados los maestros y administradores de todo el país pueden planificar y desarrollar programas de aprendizaje que sean accesibles (no simplificados) en términos de contenido de lenguaje y ciencias. Si bien no todos los maestros estarán dispuestos a aprender español, pueden utilizar estrategias tales como traducir materiales o pedir a los estudiantes que traduzcan ciertas palabras clave como apoyo (García y Kleifgen, 2010). Asimismo es esencial una exploración más profunda de las intersecciones entre conocimiento, poder y lenguaje. En este sentido, reflexionar críticamente sobre la desigual distribución social de los recursos en las escuelas (Martin y Rose, 2003) puede convertirse 
en un primer paso para repensar los programas educativos para los estudiantes refugiados en los Estados Unidos y en el mundo.

Para concluir, comprender la praxis educativa nos lleva de forma ineludible a comprender o intentar comprender todos los elementos que la constituyen como tal. A través de la historia diversos modelos de enseñanza y aprendizaje han sido diseñados, implementados y refutados. Con respecto a esto último podríamos aseverar que existe una visión que puede tornarse simplista y, en su afán de describir las modificaciones históricas en el campo de la enseñanza en diversos contextos, no advierte la evolución no lineal sino cíclica del conocimiento de las teorías de la enseñanza. Más allá de sus contradicciones han coexistido y continúan existiendo en la actualidad modelos y aplicaciones prácticas en torno a la enseñanza, proyectando un campo vasto y complejo dados todos los elementos y factores que la constituyen como tal. Estas contradicciones, evidenciadas en las ideas y las prácticas, tienen lugar en sociedades donde las nociones que quieren transmitirse encuentran en la escuela a su mejor mediador. En este sentido es importante mencionar que cada docente, en mayor o menor medida, lo haga explícito o no, basa sus prácticas didácticas en una concepción de enseñanza y aprendizaje y en una concepción del conocimiento mismo.

El objetivo de este trabajo fue explorar concepciones pedagógicas de tres educadores y también iniciar un debate en torno a las concepciones que se tejen acerca de los estudiantes refugiados e inmigrantes. Es innegable entender que cada sociedad, inscripta en un determinado momento histórico, político, cultural y económico en donde surge el saber didáctico genera las condiciones para que éste sea considerado fundado y lícito. Nuestro rol, como investigadores educativos, es entonces cuestionar, desafiar y transformar estas condiciones para mejorar las oportunidades de aprendizaje de todos los estudiantes.

\section{Referencias bibliográficas}

Administration for Children \& Families (2017). Unaccompanied Alien Children Released to Sponsors by County. Recuperado de https://www.acf.hhs.gov/orr/resource/unaccompaniedalien-children-released-to-sponsors-by-county

Callahan, R. M. \& Shifrer, D. (2016). Equitable Access for Secondary English Learner Students. Educational Administration Quarterly, 52(3), 463-496.

Cameron, D. (2001). Working with spoken discourse. Sage.

Cardozo Gaibisso, L., Allexsaht-Snider, M. \& Buxton, C. (2017). Curriculum in motion for English language learners in science: Teachers supporting newcomer unaccompanied youth. En L. de Oliveira \& K. Campbell Wilcox (Eds). Teaching Science to English Language Learners: Preparing Pre-Service and In-Service Teachers. New York: Springer, 7-29.

Cronquist, K. \& Fiszbein, A. (2017). El aprendizaje del inglés en América Latina. Recuperado de https://www.thedialogue.org/wp-content/uploads/2017/09/El-aprendizaje-del-inglésen-América-Latina-1.pdf

Davis, C. (2000). In Florida, it pays to be bilingual, University of Florida study finds. Recuperado de http://news.ufl.edu/archive/2000/01/in-florida-it-pays-to-be-bilingual-university-offlorida-study-finds.html

De Jong, E. J. \& Harper, C. A. (2011). Accommodating diversity: Pre-service teachers' views on effective practices for English language learners. Teacher preparation for linguistically diverse classrooms: A resource for teacher educators, 73-90. 
Department of Justice. (2014). Review of the President's Emergency Supplemental Request for Unaccompanied Children and Related Matters. Recuperado de https://www.gpo.gov/ fdsys/pkg/CHRG-113shrg24808/pdf/CHRG-113shrg24808.pdf

Feinberg, R. C. (2000). Newcomer schools: Salvation or segregated oblivion for immigrant students? Theory into practice, 39(4), 220-227.

Fernández, N. \& Hynes, J. (2016). The efficacy of pullout programs in elementary schools: Making it work. The Journal of Multidisciplinary Graduate Research, 2(3), 32-47.

Freeman, M. \& Vasconcelos, E. F. S. (2013). Critical social theory: Core tenets, inherent issues. New directions for evaluation, 2010(127), 7-19.

Gándara, P. (2015). With the future on the line: Why studying Latino education is so urgent. American Journal of Education, 121(3), 451-463.

García, O. \& Kleifgen, J. A. (2012). Educating emergent bilinguals: Policies, programs, and practices for English language learners. Teachers College Press.

Gee, J. (2015). Social linguistics and literacies: Ideology in discourses. Routledge.

Gooden, A. C. \& Chase, K. (2015). Habits of mind: forging university-school partnerships to bring a high-quality enrichment curriculum to English learners. VUE Voices in Urban Education. Recuperado de http://vue.annenberginstitute.org/issues/41/habits-mind-forginguniversity-school-partnerships-bring-high-quality-enrichment

Gort, M., Glenn, W. J. \& Settlage, J. (2010). Preparing teachers for linguistically diverse classrooms: A resource for teacher educators. Toward culturally and linguistically responsive teacher education: The impact of a faculty learning community on two teacher educators. Recuperado de https://experts.colorado.edu/display/pubid_229780

Guzmán, B. \& McConnell, E. D. (2002). The Hispanic population: 1990-2000 growth and change. Population Research and Policy Review, 21(1-2), 109-128.

Halliday, M. A. K. (2004). The language of science. London, UK: Continuum

Hamann, E. y Harklau, L. (2010) Education in the new Latino diaspora. En Murillo, E. (Ed.), Handbook of Latinos and education: Research, theory, and practice (157-169). Mahwah, NJ: Lawrence Erlbaum Routledge.

Hunkapiller, J. L. (2010). Impact of a Newcomer Program on Secondary School Achievement of Recent Immigrant Students (Doctoral dissertation, Texas A \& M University-Commerce). Kandel, W. (2017). Unaccompanied Alien Children: An Overview (CRS Report No. R43599 18 de enero de 2017). Congressional Research Service. Recuperado de https://fas.org/sgp/ crs/homesec/R43599.pdf

Kinslow, A. \& Sadler, T. (2018). Making science relevant. The Science Teacher, 8, 40-45.

Lemke, J. L. (1990). Talking science: language, learning, and values. Norwood, N.J.: Ablex Pub. Corp., 1990. 
Lucas, T. \& Villegas, A. M. (2013). Preparing Linguistically Responsive Teachers: Laying the Foundation in Preservice Teacher Education. En Theory Into Practice, 55-72. Ohio State University. Recuperado de https://www.researchgate.net/publication/271667021 Preparing_Linguistically_Responsive_Teachers_Laying_the_Foundation_in_Preservice Teacher Education

Martin, J. R. \& Rose, D. (2003). Working with discourse: Meaning beyond the clause. Bloomsbury Publishing.

Office of English Language Acquisition, Language Enhancement, and Academic Achievement for Limited English Proficient Students (OELA). U.S. Department of Education (2008). Biennial report to Congress on the implementation of the Title III State Formula Grant Program. School years 2004-06, Washington DC. Recuperado de https://www2.ed.gov/about/offices/list/ oela/title3biennial0406.pdf

Oikonomidoy, E. (2014). Newcomer immigrant students reinventing academic lives across national borders. Multicultural Perspectives, 16(3), 141. doi:10.1080/15210960.2014.922882

Patel, S. G., Tabb, K. M., Strambler, M. J. \& Eltareb, F. (2015). Newcomer immigrant adolescents and ambiguous discrimination: The role of cognitive appraisal. Journal of Adolescent Research, 30(1), 7-30.

Portes, P. R. \& Salas, S. (2015). Nativity shifts, broken dreams, and the new Latino South's Post-First Generation. Peabody Journal of Education, 90(3), 426-436.

Rogers, R. (Ed.). (2011). An introduction to critical discourse analysis in education. Routledge.

Ruona, W. E. (2005). Analyzing qualitative data. Research in organizations: Foundations and methods of inquiry, 223, 263.

Seghetti, L., Siskin, A. \& Wasem, R. (2015). Unaccompanied alien children: An overview. En C. A. Toller (Ed.), Central America's gang violence and the rise of unaccompanied children in the U.S. (31-52). New York, NY: Nova Science Publishers.

Sleeter, C. E. (2001). Preparing teachers for culturally diverse schools research and the overwhelming presence of whiteness. Journal of teacher education, 52(2), 94-106.

Terrio, S. (2015). Whose child am I?: Unaccompanied, undocumented children in US immigration custody. Oakland, CA: University of California Press

Wodak, R. (2009). The discourse of politics in action: politics as usual. Basingstoke, England: Palgrave Macmillan. Recuperado de https://www.researchgate.net/publication/263561029 Ruth_Wodak_The_Discourse_of_Politics_in_Action_Politics_as_Usual

WSBTV (2014). 28 Hall Co. Students were reportedly unaccompanied minor immigrants. Recuperado de http://www.wsbtv.com/news/local/28-hall-county-students-werereportedly-unaccompan/137584856 